\title{
A Non-Stationary MIMO Vehicle-to-Vehicle Channel Model Derived From the Geometrical T-Junction Model
}

\author{
Ali Chelli and Matthias Pätzold \\ University of Agder \\ Norway
}

\section{Introduction}

According to the European commission (Road Safety Evolution in EU, 2009), 1.2 million road accidents took place in the European Union in 2007. These road accidents have resulted in 1.7 million injuries and more than 40 thousand deaths. It turned out that human errors were involved in $93 \%$ of these accidents. V2V communication is a key element in reducing road casualties. For the development of future V2V communication systems, the exact knowledge of the statistics of the underlying fading channel is necessary. Several channel models for $\mathrm{V} 2 \mathrm{~V}$ communications can be found in the literature. For example, the two-ring channel model for V2V communications has been presented in (Pätzold et al., 2008). There, a reference and a simulation model have been derived starting from the geometrical two-ring model. In (Zajić et al., 2009), a three-dimensional reference model for wideband MIMO V2V channels has been proposed. The model takes into account single-bounce and double-bounce scattering in vehicular environments. The geometrical street model (Chelli \& Pätzold, 2008) captures the propagation effects if the communicating vehicles are moving along a straight street with local roadside obstructions (buildings, trees, etc.). In (Acosta et al., 2004), a statistical frequency-selective channel model for small-scale fading is presented for a V2V communication links.

The majority of channel models that can be found in the literature rely on the stationarity assumption. However, measurement results for V2V channels in (Paier et al., 2008) have shown that the stationarity assumption is valid only for very short time intervals. This fact arises the need for non-stationary channel models. Actually, if the communicating cars are moving with a relatively high speed, the AoD and the AoA become time-variant resulting in a non-stationary channel model. The traditional framework invoked in case of stationary stochastic processes cannot be used to study the statistical properties of non-stationary channels. In the literature, quite a few time-frequency distributions have been proposed to study non-stationary deterministic signals (Cohen, 1989). A review of these distributions can be found in (Cohen, 1989). Many commonly used time-frequency distributions are members of the Cohen class (O'Neill \& Williams, 1999). It has been stated in (Sayeed \& Jones, 1995) that the Cohen class, although introduced for deterministic signals, can be applied on non-stationary stochastic processes. 
In this chapter, we present a non-stationary MIMO V2V channel model. The AoD and the AoA are supposed to be time dependent. This assumption makes our channel model non-stationary. The correlation properties of a non-stationary channel model can be obtained using a multi-window spectrogram (Paier et al., 2008). For rapidly changing spectral content however, finding an appropriate time window size is a rather complicated task. The problem is that a decrease in the time window size improves the time resolution, but reduces the frequency resolution. To overcome this problem, we make use of the Choi-Williams distribution proposed in (Choi \& Williams, 1989). The extremely non-isotropic propagation environment is modelled using the T-junction scattering model (Zhiyi et al., 2009). In contrast to the original multi-cluster T-model, we assume to simplify matters that each cluster consists of only one scatterer. Under this assumption, the reference and the simulation model are identical. The main contribution of this chapter is that it presents a non-stationary channel model with time-variant AoD and AoA. Moreover, analytical expressions for the correlation properties of the non-stationary channel model are provided, evaluated numerically, and then illustrated.

The rest of the chapter is organized as follows. In Section 2, the geometrical T-model is presented. Based on this geometrical model, we derive a reference (simulation) model in Section 3. In Section 4, the correlation properties of the proposed channel model are studied. Numerical results of the correlation functions are presented in Section 5. Finally, we draw the conclusions in Section 6.

\section{The Geometrical T-junction Model}

A typical propagation scenario for $\mathrm{V} 2 \mathrm{~V}$ communications at a $\mathrm{T}$-junction is presented in Fig. 1. Fixed scatterers are located on both sides of the T-junction. In order to derive the statistical properties of the corresponding MIMO V2V channel, we first need to find a geometrical model that describes properly the vehicular T-junction propagation environment. This geometrical model is illustrated in Fig. 2. It takes into account double-bounce scattering under non-line-of sight conditions. Each building is modelled by one scatterer which makes our model extremely non-isotropic. The scatterers in the neighborhood of the transmitter $\mathrm{MS}_{T}$ are denoted by $S_{m}^{T}(m=1,2, \ldots, M)$, whereas the scatterers close to the receiver $\mathrm{MS}_{R}$ are designated by $S_{n}^{R}(n=1,2, \ldots, N)$. The total number of scatterers near to the transmitter is denoted by $N$, while the total number of scatterers near to the receiver is designated by $M$. The transmitter and the receiver are moving towards the intersection point with the velocities $\mathrm{v}_{T}$ and $\mathrm{v}_{R}$, respectively. The direction of motions of the transmitter and the receiver w.r.t. the $x$-axis are referred to as $\phi_{T}$ and $\phi_{R}$, respectively. The AoD are time-variant and are denoted by $\alpha_{m}^{T}(t)$, while the symbol $\beta_{n}^{R}(t)$ stands for the AoA. The AoD and the AoA are independent since double-bounce scattering is assumed. The transmitter and the receiver are equipped with an antenna array encompassing $M_{T}$ and $M_{R}$ antenna elements, respectively. The antenna element spacing at the transmitter side is denoted by $\delta_{T}$. Analogously, the antenna element spacing at the receiver side is referred to as $\delta_{R}$. The tilt angle of the transmit antenna array is denoted by $\gamma_{T}$, while $\gamma_{R}$ stands for the tilt angle for the receive antenna array. The transmitter (receiver) is located at a distance $h_{1}^{T}\left(h_{1}^{R}\right)$ from the left-hand side of the street and at a distance $h_{2}^{T}\left(h_{2}^{R}\right)$ from the right-hand side seen in moving direction. 


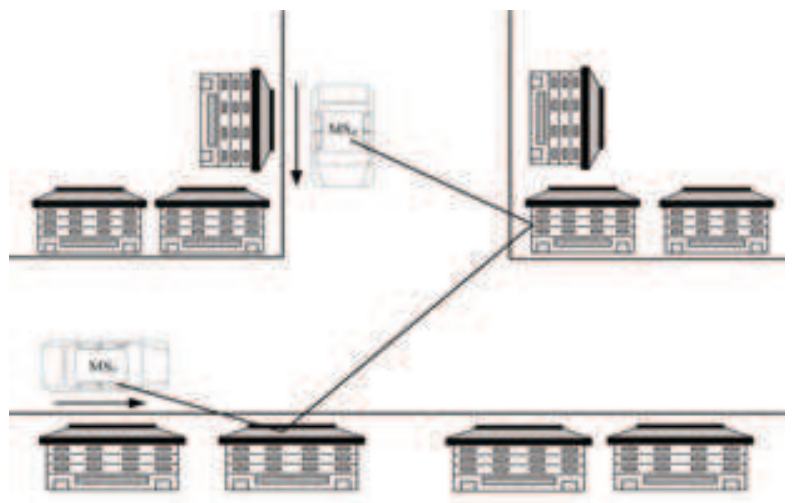

Fig. 1. Typical propagation scenario for V2V communications at a T-junction.

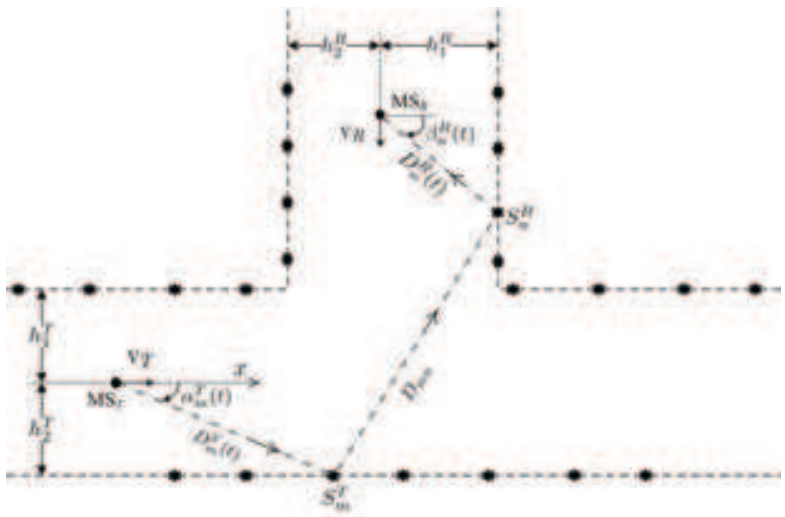

Fig. 2. The geometrical T-Junction model for V2V communications.

\section{The Reference Model}

The starting point for the derivation of the reference model for the MIMO V2V channel is the geometrical T-junction model presented in Fig. 2. For the reference model, we assume double-bounce scattering from fixed scatterers. We distinguish between the scatterers near to the transmitter and the scatterers close to the receiver. It can be seen from Fig. 2 that a wave emitted from the $l$ th transmit antenna element $A_{l}^{T}\left(l=1,2, \ldots, M_{T}\right)$ travels over the scatterers $S_{m}^{T}$ and $S_{n}^{R}$ before impinging on the $k$ th receive antenna element $A_{k}^{R}\left(k=1,2, \ldots, M_{R}\right)$. Using the wave propagation model in (Pätzold et al., 2008), the complex channel gain $g_{k l}\left(\vec{r}_{T}, \vec{r}_{R}\right)$ describing the link $A_{l}^{T}-A_{k}^{R}$ of the underlying $M_{T} \times M_{R}$ MIMO V2V channel model can be expressed in the present case as

$$
g_{k l}\left(\vec{r}_{T}, \vec{r}_{R} \neq \sum_{m=1, n=1}^{M, N} c_{m n} e^{j\left(\theta_{m n}(t)+\vec{k}_{m}^{T} \cdot \vec{r}_{T}-\vec{k}_{n}^{R} \cdot \vec{r}_{R}-k_{0} d_{m n}(t)\right) .}\right.
$$


The symbols $c_{m n}$ and $\theta_{m n}(t)$ stand for the the joint gain and the joint phase shift caused by the scatterers $S_{m}^{T}$ and $S_{n}^{R}$. The joint channel gain can be written as $c_{m n}=1 / \sqrt{M N}$ (Pätzold et al., 2008). The phase shift $\theta_{m n}(t)$ is a stochastic process, as the $\operatorname{AoD} \alpha_{m}^{T}(t)$ and the AoA $\beta_{n}^{R}(t)$ are time-variant. This is in contrast to the models proposed in (Pätzold et al., 2008) and (Zhiyi et al., 2009), where the phase shift is a random variable. The joint phase shift can be expressed as $\theta_{m n}(t)=\left(\theta_{m}(t)+\theta_{n}^{\prime}(t)\right) \bmod 2 \pi$, where $\bmod$ stands for the modulo operation. The terms $\theta_{m}(t)$ and $\theta_{n}^{\prime}(t)$ are the phase shifts associated with the scatterers $S_{m}^{T}$ and $S_{n}^{R}$, respectively.

The second phase term in (1), $\vec{k}_{m}^{T} \cdot \vec{r}_{T}$, is caused by the movement of the transmitter. The wave vector pointing in the propagation direction of the $m$ th transmitted plane wave is denoted by $\vec{k}_{m}^{T}$, while $\vec{r}_{T}$ stands for the spatial translation vector of the transmitter. The scalar product $\vec{k}_{m}^{T} \cdot \vec{r}_{T}$ can be expanded as

$$
\vec{k}_{m}^{T} \cdot \vec{r}_{T}=2 \pi f_{\max }^{T} \cos \left(\alpha_{m}^{T}(t)-\phi_{T}\right) t
$$

where $f_{\max }^{T}=\mathrm{v}_{T} / \lambda$ denotes the maximum Doppler frequency associated with the mobility of the transmitter. The symbol $\lambda$ refers to the wavelength. The time-variant $\operatorname{AoD} \alpha_{m}^{T}(t)$ can be expressed as

$$
\alpha_{m}^{T}(t)=\left\{\begin{array}{llc}
-\pi+g_{2}(t) & \text { if } & -\pi \leq \alpha_{m}^{T}(t) \leq-\frac{\pi}{2} \\
g_{2}(t) & \text { if } & -\frac{\pi}{2}<\alpha_{m}^{T}(t) \leq 0 \\
g_{1}(t) & \text { if } & 0<\alpha_{m}^{T}(t) \leq \frac{\pi}{2} \\
\pi+g_{1}(t) & \text { if } & \frac{\pi}{2}<\alpha_{m}^{T}(t) \leq \pi
\end{array}\right.
$$

where

$$
\begin{aligned}
& g_{1}(t)=\arctan \left(\frac{h_{1}^{T} \tan \left(\alpha_{m}^{T}\left(t_{0}\right)\right)}{h_{1}^{T}-\mathrm{v}_{T}\left(t-t_{0}\right) \tan \left(\alpha_{m}^{T}\left(t_{0}\right)\right)}\right) \\
& g_{2}(t) \arctan \left(\frac{h_{2}^{T} \tan \left(\alpha_{m}^{T}\left(t_{0}\right)\right)}{h_{2}^{T}-\mathrm{v}_{T}\left(t-t_{0}\right) \tan \left(\alpha_{m}^{T}\left(t_{0}\right)\right)}\right) .
\end{aligned}
$$

We assume that the AoD seen from the transmitter side can be considered as constant for a given time interval if the angular deviation does not exceed a certain threshold. For instance, the $\operatorname{AoD} \alpha_{m}^{T}\left(t_{1}\right)$ at time instant $t_{1}$ and the $\operatorname{AoD} \alpha_{m}^{T}\left(t_{2}\right)$ at time instant $t_{2}$ are equal if the angle difference $\left|\alpha_{m}^{T}\left(t_{1}\right)-\alpha_{m}^{T}\left(t_{2}\right)\right| \leq \epsilon_{\alpha}$, with $\epsilon_{\alpha}$ is a very small positive value. In this way, the AoD $\alpha_{m}^{T}(t)$ can be written as

$$
\alpha_{m}^{T}(t) \alpha_{m, i-1}^{T} \text { if } t_{i-1} \leq t<t_{i} \text { for } i=1,2, \ldots
$$

The term $\alpha_{m, i-1}^{T}$ is a constant that can be obtained from (3) by setting the time $t$ to $t_{i-1}$. The length of the intervals $\left[t_{i-1}, t_{i}\right)$ and $\left[t_{i}, t_{i+1}\right)$ can be quite different for $i=1,2, \ldots$. The phase shift introduced by a scatterer is generally dependent on the direction of the outgoing wave. Hence, a change in the $\operatorname{AoD} \alpha_{m}^{T}(t)$ results in a new random phase shift. Since the AoD $\alpha_{m}^{T}(t)$ is defined piecewise, the phase shift $\theta_{m}(t)$ is also defined piecewise as follows

$$
\theta_{m}(t) \theta_{m, i-1} \text { if } t_{i-1} \leq t<t_{i} \text { for } i=1,2, \ldots
$$


where $\theta_{m, 0}, \theta_{m, 1}, \ldots$ are independent identically distributed (i.i.d.) random variables uniformly distributed over $[0,2 \pi)$.

The third phase term in (1), $\vec{k}_{n}^{R} \cdot \vec{r}_{R}$, is associated with the movement of the receiver. The symbol $\vec{k}_{n}^{R}$ stands for the wave vector pointing in the propagation direction of the $n$th received plane wave, while $\vec{r}_{R}$ represents the spatial translation vector of the receiver. The scalar product $\vec{k}_{n}^{R} \cdot \vec{r}_{R}$ can be expanded as

$$
\vec{k}_{n}^{R} \cdot \vec{r}_{R}=-2 \pi f_{\max }^{R} \cos \left(\beta_{n}^{R}(t)-\phi_{R}\right) t
$$

where $f_{\max }^{R}=\mathrm{v}_{R} / \lambda$ denotes the maximum Doppler frequency caused by the receiver movement. Using the geometrical T-junction model shown in Fig. 2, the time-variant AoA $\beta_{n}^{R}(t)$ can be expressed as

$$
\beta_{n}^{R}(t)=\left\{\begin{array}{llc}
-\pi+g_{3}(t) & \text { if } & -\pi \leq \beta_{n}^{R}(t) \leq-\frac{\pi}{2} \\
g_{4}(t) & \text { if } & -\frac{\pi}{2}<\beta_{n}^{R}(t) \leq \frac{\pi}{2} \\
\pi+g_{3}(t) & \text { if } & \frac{\pi}{2}<\beta_{n}^{R}(t) \leq \pi
\end{array}\right.
$$

where

$$
\begin{aligned}
& g_{3}\left(\operatorname{tanctan}\left(\frac{h_{2}^{R} \tan \left(\beta_{n}^{R}\left(t_{0}\right)\right)-\mathrm{v}_{R}\left(t-t_{0}\right)}{h_{2}^{R}}\right)\right. \\
& g_{4}\left(\operatorname{tarctan}\left(\frac{h_{1}^{R} \tan \left(\beta_{n}^{R}\left(t_{0}\right)\right)-\mathrm{v}_{R}\left(t-t_{0}\right)}{h_{1}^{R}}\right) .\right.
\end{aligned}
$$

We assume that the AoA seen from the receiver side can be considered as constant for a given time interval if the angular deviation does not exceed a certain threshold. For instance, the AoA $\beta_{n}^{R}\left(t_{1}^{\prime}\right)$ at time instant $t_{1}^{\prime}$ and the AoA $\beta_{n}^{R}\left(t_{2}^{\prime}\right)$ at time instant $t_{2}^{\prime}$ are equal if the angle difference $\left|\beta_{n}^{R}\left(t_{1}^{\prime}\right)-\beta_{n}^{R}\left(t_{2}^{\prime}\right)\right| \leq \epsilon_{\alpha}$. In this way, the AoA $\beta_{n}^{R}(t)$ can be written as

$$
\beta_{n}^{R}(t) \beta_{n, j-1}^{R} \text { if } t_{j-1}^{\prime} \leq t<t_{j}^{\prime} \text { for } j=1,2, \ldots
$$

The term $\beta_{n, j-1}^{R}$ is a constant that can be obtained from (9) by setting the time $t$ to $t_{j-1}^{\prime}$. The length of the intervals $\left[t_{j-1}^{\prime}, t_{j}^{\prime}\right)$ and $\left[t_{j}^{\prime}, t_{j+1}^{\prime}\right)$ can be quite different for $j=1,2, \ldots$. The phase shift introduced by a scatterer is generally dependent on the direction of the incoming wave. Hence, a change in the AoA $\beta_{n}^{R}(t)$ results in a new random phase shift. Since the AoA $\beta_{n}^{R}(t)$ is defined piecewise, the phase shift $\theta_{n}^{\prime}(t)$ is also defined piecewise as follows

$$
\theta_{n}^{\prime}(t) \theta_{n, j-1}^{\prime} \text { if } t_{j-1}^{\prime} \leq t<t_{j}^{\prime} \text { for } j=1,2, \ldots
$$

where $\theta_{n, 0}^{\prime}, \theta_{n, 1}^{\prime}, \ldots$ are i.i.d. random variables uniformly distributed over $[0,2 \pi)$. After substituting (2) and (8) in (1), the complex channel gain $g_{k l}(t)$ can be expressed as

$$
g_{k l}(t)=\sum_{m, n=1}^{M, N} \frac{a_{m}^{T} b_{n}^{R} c_{m n}^{T R}}{\sqrt{M N}} e^{j\left(2 \pi\left(f_{m}^{T}+f_{n}^{R}\right) t+\theta_{m n}(t)\right)}
$$


where

$$
\begin{aligned}
a_{m}^{T} & =e^{j \pi \frac{\delta_{T}}{\lambda}\left(M_{T}-2 l+1\right) \cos \left(\alpha_{m}^{T}(t)-\gamma_{T}\right)} \\
b_{n}^{R} & =e^{j \pi \frac{\delta_{R}}{\lambda}\left(M_{R}-2 k+1\right) \cos \left(\beta_{n}^{R}(t)-\gamma_{R}\right)} \\
c_{m n}^{T R} & =e^{-j \frac{2 \pi}{\lambda}\left(D_{m}^{T}(t)+D_{m n}+D_{n}^{R}(t)\right)} \\
f_{m}^{T} & =f_{\max }^{T} \cos \left(\alpha_{m}^{T}(t)-\phi^{T}\right) \\
f_{n}^{R} & =f_{\max }^{R} \cos \left(\beta_{n}^{R}(t)-\phi^{R}\right) .
\end{aligned}
$$

with $D_{m}^{T}(t)$ denoting the distance from the transmitter to the scatterer $S_{m}^{T}$. The term $D_{m n}$ represents the distance between the scatterers $S_{m}^{T}$ and $S_{n}^{R}$, while $D_{n}^{R}(t)$ corresponds to the distance from the receiver to the scatterer $S_{n}^{R}$, as shown in Fig. 2.

\section{Correlation Properties}

For wide-sense stationary processes, the temporal ACF depends only on the time difference $\tau$. However, for non-stationary processes, the temporal ACF does not only depend on the time difference $\tau$, but also on the time $t$. Due to its time dependance, the ACF of non-stationary processes is called local ACF (Cohen, 1989). Several definitions for the local ACF have been proposed in literature. In this paper, we utilize the definition of the local ACF proposed by Wigner (Cohen, 1989), which is given by

$$
r_{g_{k l}}(t, \tau):=E\left\{g_{k l}(t+\tau / 2) g_{k l}^{*}(t-\tau / 2)\right\}
$$

where $(\cdot)^{*}$ denotes the complex conjugation and $E\{\cdot\}$ stands for the expectation operator. By applying the expectation operator on the i.i.d. random variables $\theta_{m, i}(i=0,1, \ldots)$ and $\theta_{n, j}^{\prime}$ $(j=0,1, \ldots)$ and exploiting their independence, we can express the local ACF as

$$
r_{g_{k l}}(t, \tau)=r_{g_{k l}}^{T}(t, \tau) \cdot r_{g_{k l}}^{R}(t, \tau)
$$

where

$$
\begin{aligned}
& r_{g_{k l}}^{T}\left(t, \tau=\frac{1}{M} \sum_{m=1}^{M} e^{j 2 \pi\left(f_{m}^{T}\left(t+\frac{\tau}{2}\right)\left(t+\frac{\tau}{2}\right)-f_{m}^{T}\left(t-\frac{\tau}{2}\right)\left(t-\frac{\tau}{2}\right)\right)}\right. \\
& r_{g_{k l}}^{R}\left(t, \tau=\frac{1}{N} \sum_{n=1}^{N} e^{j 2 \pi\left(f_{n}^{R}\left(t+\frac{\tau}{2}\right)\left(t+\frac{\tau}{2}\right)-f_{n}^{R}\left(t-\frac{\tau}{2}\right)\left(t-\frac{\tau}{2}\right)\right)} .\right.
\end{aligned}
$$

Note that the local ACF $r_{g_{k l}}(t, \tau)$ is written as a product of the local transmit $\operatorname{ACF} r_{g_{k l}}^{T}(t, \tau)$ and the local receive ACF $r_{g_{k l}}^{T}(t, \tau)$ since we assume a limited number of scatterers in the proposed model. The expression of the local ACF is derived using the Wigner method (Cohen, 1989). By applying the Fourier transformation on the local ACF in (21), we obtain the Wigner time-frequency distribution. The former even though a member of the Cohen class of distributions, suffers from the cross-term problem (Cohen, 1989). To deal with this issue, a kernel function aiming to reduce the cross-terms need to be introduced. One of the effective distributions in diminishing the effect of cross-terms is the Choi-Williams distribution (Choi \& Williams, 1989). Choi and Williams devised their kernel function in such a way that a relatively large weight is given to $g_{k l}(u+\tau / 2) g_{k l}^{*}(u-\tau / 2)$ if $u$ is close to $t$. In this way, they 
emphasis the local behaviour of the channel and guarantee that the non-stationarities will not be smeared in time and frequency. The kernel function for the Choi-Williams distribution is given by $\phi(\xi, \tau)=e^{-\xi^{2} \tau^{2} / \sigma}$. It follows that the generalized local ACF can be expressed as (Choi \& Williams, 1989)

$$
\begin{aligned}
K(t, \tau ; \phi) & =\int_{-\infty}^{\infty} \int_{-\infty}^{\infty} e^{j 2 \pi \xi(u-t)} \phi(\xi, \tau) r_{g_{k l}}(u, \tau) d u d \xi \\
& =\int_{-\infty}^{\infty} \frac{r_{g_{k l}}(u, \tau)}{\sqrt{4 \pi \tau^{2} / \sigma}} \exp \left(-\frac{(u-t)^{2}}{4 \tau^{2} / \sigma}\right) d u .
\end{aligned}
$$

The generalized local ACF presented above can be used for both stationary and non-stationary processes. Actually, if the process is stationary, the local ACF $r_{g_{k l}}(u, \tau)$ equals $r_{g_{k l}}(\tau)$. Using (24), it turns out that the generalized local ACF for stationary processes equals the classical ACF, i.e., $K(t, \tau ; \phi)=r_{g_{k l}}(\tau)$.

For stationary processes, the power spectral density can be obtained from the Fourier transformation of the temporal ACF. Analogously, for non-stationary processes, the time-frequency distribution can be obtained from the generalized local ACF by applying the Fourier transformation. The time-frequency distribution gives an insight into how the power spectrum varies with time $t$. The time-frequency distribution $W(t, f ; \phi)$ can be written as

$$
\begin{aligned}
W(t, f ; \phi & \Rightarrow \int_{-\infty}^{\infty} K(t, \tau ; \phi) e^{-j 2 \pi f \tau} d \tau \\
& =\int_{-\infty}^{\infty} \int_{-\infty}^{\infty} A(\xi, \tau ; \phi) e^{-j 2 \pi(f \tau-t \xi)} d \tau d \xi
\end{aligned}
$$

where $A(\xi, \tau ; \phi)$ is the ambiguity function.

The local space CCF can be expressed as

$$
\begin{aligned}
& \rho_{k l, k^{\prime} l^{\prime}}\left(t, \delta_{T}, \delta_{R} \text { F }\left\{g_{k l}(t) g_{k^{\prime} l^{\prime}}^{*}(t)\right\}\right. \\
&=\frac{1}{M} \sum_{m=1}^{M} e^{-j 2 \pi \frac{\delta_{T}}{\lambda}\left(l-l^{\prime}\right) \cos \left(\alpha_{m}^{T}(t)-\gamma_{T}\right)} \\
& \cdot \frac{1}{N} \sum_{n=1}^{N} e^{-j 2 \pi \frac{\delta_{R}}{\lambda}\left(k-k^{\prime}\right) \cos \left(\beta_{n}^{R}(t)-\gamma_{R}\right)} \\
&= \rho_{l l^{\prime}}^{T}\left(t, \delta_{T}\right) \cdot \rho_{k k^{\prime}}^{R}\left(t, \delta_{R}\right) .
\end{aligned}
$$

In (26), the $\operatorname{AoD} \alpha_{m}^{T}(t)$ and the $\operatorname{AoA} \beta_{n}^{R}(t)$ are given by (6) and (12), respectively. Note that the local space CCF is written as a product of the local transmit space correlation function $(\mathrm{CF})$ $\rho_{l l^{\prime}}^{T}\left(t, \delta_{T}\right)$ and the local receive space CF $\rho_{k k^{\prime}}^{R}\left(t, \delta_{R}\right)$.

\section{Numerical Results}

In this section, the analytical expressions presented in the previous section are evaluated numerically and then illustrated. The propagation environment encompasses twelve scatterers around the transmitter. Six scatterers are located on the left side of the transmitter and the remaining scatterers are on the right side. The distance between two successive scatterers is set to $20 \mathrm{~m}$. We consider the same number of scatterers around the receiver. The transmitter and the receiver have a velocity of $70 \mathrm{~km} / \mathrm{h}$ and a direction of motion determined 
by $\phi_{T}=0$ and $\phi_{R}=-\pi / 2$, respectively. The transmitter and the receiver antenna tilt angles $\gamma_{T}$ and $\gamma_{R}$ are equal to $\pi / 2$. The street parameters are chosen as $h_{1}^{T}=h_{2}^{T}=50 \mathrm{~m}$ and $h_{1}^{R}=h_{2}^{R}=50 \mathrm{~m}$. The parameter $\epsilon_{\alpha}$ is set to 0.1 .

The absolute value of the resulting generalized local ACF $K(t, \tau ; \phi)$ is illustrated in Fig. 3 . From this figure, we can see that the shape of the local ACF changes for different values of $t$, which is due to the non-stationarity of the channel model. If the channel model is stationary, we would observe the same shape of the local ACF at different time instants $t$. The absolute value of the time-frequency distribution, $|W(t, f ; \phi)|$, shown in Fig. 4, is obtained from the generalized local ACF by applying the Fourier transform w.r.t. the time lag $\tau$. It can be seen from this figure how the Doppler spectrum of the channel varies with time $t$. For the chosen propagation scenario, it can be observed from Fig. 4 that the zero Doppler frequency has the highest power for all time instants $t$. The power of the non-zero Doppler frequencies decays for a certain period of time before increasing again. The absolute value of the local transmit space CF $\left|\rho_{12}^{T}\left(t, \delta_{T}\right)\right|$ is presented in Fig. 5. It can be seen from this figure that the amplitude of this function is more sensitive to the transmit antenna spacing $\delta_{T}$ than to the time $t$. The absolute value of the local receive space $\mathrm{CF}\left|\rho_{12}^{R}\left(t, \delta_{R}\right)\right|$ is illustrated in Fig. 6. For the chosen scenario, this function decays faster than the local transmit space $C F$ w.r.t. the antenna spacing.

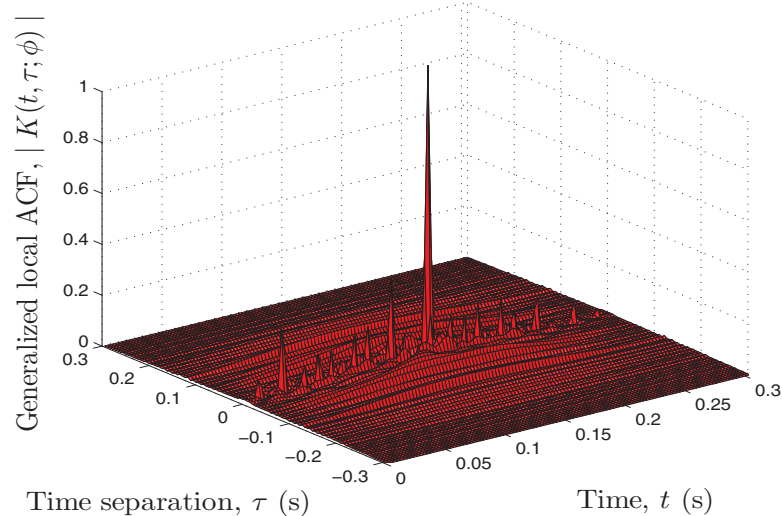

Fig. 3. The absolute value of the generalized local ACF $|K(t, \tau ; \phi)|$.

\section{Conclusion}

In this chapter, we have presented a non-stationary MIMO V2V channel model. Based on the geometrical T-junction model, we have derived an expression for the time-variant channel gain taking into account double-bounce scattering from fixed scatterers. We have assumed a limited number of scatterers. Under this assumption, the reference model equals the simulation model. In vehicular environments, the high speed of the communicating vehicles results in time-variant $\mathrm{AoD}$ and $\mathrm{AoA}$. This property is taken into account in our channel model, which makes the model non-stationary. To study the statistical properties of the proposed channel model, we utilized the Choi-Williams distribution. We have provided analytical expressions of the generalized local ACF, the time-frequency distribution, and the local space CCF. The latter can be written as a product of the local transmit space CF and 


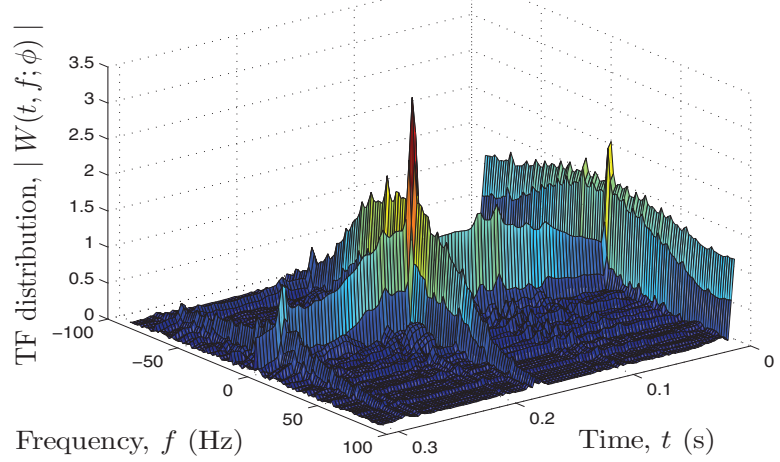

Fig. 4. The absolute value of the time-frequency distribution $|W(t, f ; \phi)|$.

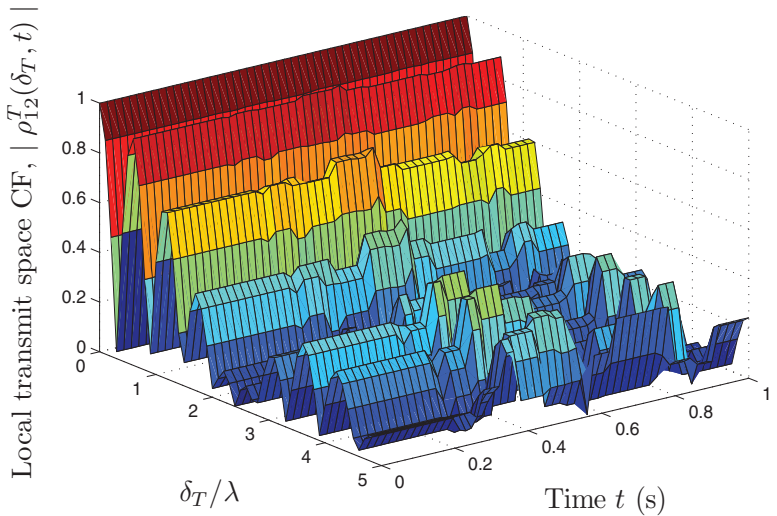

Fig. 5. Absolute value of the local transmit space CF $\left|\rho_{12}^{T}\left(t, \delta_{T}\right)\right|$.

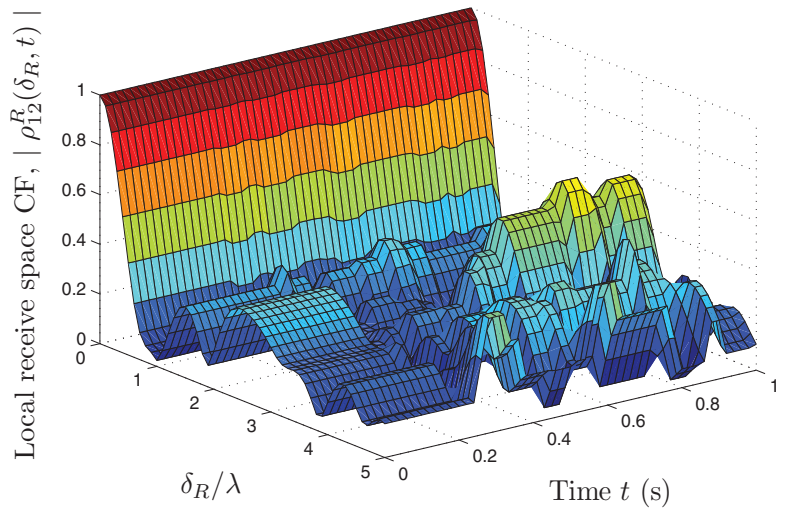

Fig. 6. Absolute value of the local receive space $\mathrm{CF}\left|\rho_{12}^{R}\left(t, \delta_{R}\right)\right|$. 
the local receive space CF. Supported by our analysis, we can conclude that the stationarity assumption is violated for $\mathrm{V} 2 \mathrm{~V}$ channels, especially if the mobile speed is high and the observation interval is large. Non-stationary channel models are needed as a tool for designing future V2V communication systems. In future work, the effect of moving scatterers on the channel statistics will be studied.

\section{References}

Acosta, G., Tokuda, K. \& Ingram, M. (2004). Measured joint doppler-delay power profiles for vehicle-to-vehicle communications at $2.4 \mathrm{GHz}$, Proc. IEEE Global Telecommunications Conference, 2004. GLOBECOM '04., Vol. 6, Dallas, TX, pp. 3813-3817.

Chelli, A. \& Pätzold, M. (2008). A wideband multiple-cluster MIMO mobile-to-mobile channel model based on the geometrical street model, Proc. 19th IEEE International Symposium on Personal, Indoor and Mobile Radio Communications, PIMRC 2008, Cannes, France, pp. 1-6.

Choi, H.-I. \& Williams, W. (1989). Improved time-frequency representation of multicomponent signals using exponential kernels, IEEE Transactions on Acoustics, Speech, and Signal Processing 37(6): 862-871.

Cohen, L. (1989). Time-frequency distributions-a review, Proceedings of the IEEE 77(7): 941-981.

O'Neill, J. \& Williams, W. (1999). Shift covariant time-frequency distributions of discrete signals, IEEE Transactions on Signal Processing 47(1): 133-146.

Paier, A., Zemen, T., Bernado, L., Matz, G., Karedal, J., Czink, N., Dumard, C., Tufvesson, F., Molisch, A. F. \& Mecklenbräuker, C. F. (2008). Non-WSSUS vehicular channel characterization in highway and urban scenarios at $5.2 \mathrm{GHz}$ using the local scattering function, Proc. 2008 International ITG Workshop on Smart Antennas (WSA 2008), Darmstadt, Germany, pp. 9-15.

Pätzold, M., Hogstad, B. \& Youssef, N. (2008). Modeling, analysis, and simulation of MIMO mobile-to-mobile fading channels, IEEE Transactions on Wireless Communications $7(2): 510-520$.

Road Safety Evolution in EU (2009). Technical report, European Commision/ Directorate General Energy Transport.

Sayeed, A. \& Jones, D. (1995). Optimal kernels for nonstationary spectral estimation, IEEE Transactions on Signal Processing 43(2): 478-491.

Zajić, A. G., Stüber, G. L., Pratt, T. G. \& Nguyen, S. T. (2009). Wideband MIMO mobile-to-mobile channels: geometry-based statistical modeling with experimental verification, IEEE Transactions on Vehicular Technology 58(2): 517-534.

Zhiyi, H., Wei, C., Wei, Z., Pätzold, M. \& Chelli, A. (2009). Modelling of MIMO vehicle-to-vehicle fading channels in T-junction scattering environments, $3 r d$ European Conference on Antennas and Propagation, 2009. EuCAP 2009., Berlin, Germany, pp. 652-656. 


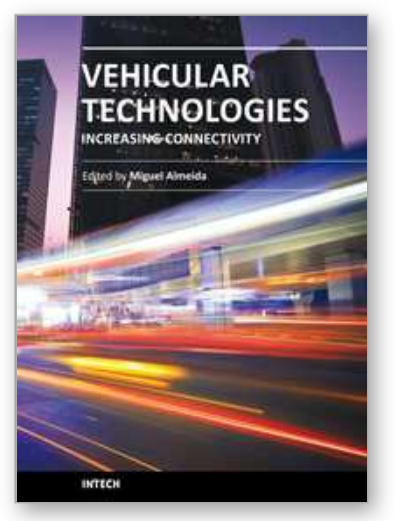

\author{
Vehicular Technologies: Increasing Connectivity \\ Edited by Dr Miguel Almeida
}

ISBN 978-953-307-223-4

Hard cover, 448 pages

Publisher InTech

Published online 11, April, 2011

Published in print edition April, 2011

This book provides an insight on both the challenges and the technological solutions of several approaches, which allow connecting vehicles between each other and with the network. It underlines the trends on networking capabilities and their issues, further focusing on the MAC and Physical layer challenges. Ranging from the advances on radio access technologies to intelligent mechanisms deployed to enhance cooperative communications, cognitive radio and multiple antenna systems have been given particular highlight.

\title{
How to reference
}

In order to correctly reference this scholarly work, feel free to copy and paste the following:

Ali Chelli and Matthias Pätzold (2011). A Non-Stationary MIMO Vehicle-to-Vehicle Channel Model Derived From the Geometrical T-Junction Model, Vehicular Technologies: Increasing Connectivity, Dr Miguel Almeida (Ed.), ISBN: 978-953-307-223-4, InTech, Available from: http://www.intechopen.com/books/vehiculartechnologies-increasing-connectivity/a-non-stationary-mimo-vehicle-to-vehicle-channel-model-derived-fromthe-geometrical-t-junction-model

\section{INTECH}

open science | open minds

\section{InTech Europe}

University Campus STeP Ri

Slavka Krautzeka 83/A

51000 Rijeka, Croatia

Phone: +385 (51) 770447

Fax: +385 (51) 686166

www.intechopen.com

\section{InTech China}

Unit 405, Office Block, Hotel Equatorial Shanghai

No.65, Yan An Road (West), Shanghai, 200040, China

中国上海市延安西路65号上海国际贵都大饭店办公楼 405 单元

Phone: +86-21-62489820

Fax: +86-21-62489821 
(C) 2011 The Author(s). Licensee IntechOpen. This chapter is distributed under the terms of the Creative Commons Attribution-NonCommercialShareAlike-3.0 License, which permits use, distribution and reproduction for non-commercial purposes, provided the original is properly cited and derivative works building on this content are distributed under the same license. 